Surface Science 99 (1980) 59-69

(c) North-Holland Publishing Company

\title{
REFLECTOMETRIC STUDY OF SURFACE STATES AND OXYGEN ADSORPTION ON CLEAN Si(100) AND (110) SURFACES
}

\author{
P.E. WIERENGA, M.J. SPARNAAY AND A. VAN SILFHOUT \\ Twente University of Technology, Depurtment of Applied Physics, 7500 AE Enschede, The \\ Netherlands
}

Received 26 November 1979 ; accepted for publication 11 February 1980

External differential reflection measurements were carried out on clean Si(100) and (110) surfaces in the photon energy range of 1.0 to $3.0 \mathrm{eV}$ at 300 and $80 \mathrm{~K}$. The results for $\mathrm{Si}(100)$ at $300 \mathrm{~K}$ showed two peaks in the joint density of states curve, which sharpened at $80 \mathrm{~K}$. One peak at $3.0 \pm 0.2 \mathrm{eV}$ can be attributed to optical transitions from a filled surface states band near the top of the valence band to empty bulk conduction band levels. The other peak at $1.60 \pm 0.05 \mathrm{eV}$ may be attributed to transitions to an empty surface states band in the energy gap. This result favours the asymmetric dimer model for the $\mathrm{Si}(100)$ surface. For the (110) surface at $300 \mathrm{~K}$ only one peak was found at $3.0 \pm 0.2 \mathrm{eV}$. At $80 \mathrm{~K}$ the peak height diminished by a factor of two. Oxygen adsorption in the submonolayer region on the clean Si(100) surface appeared to proceed in a similar way as on the Si(111) $7 \times 7$ surface. For the Si(110) surface the kinetics of the adsorption process at $80 \mathrm{~K}$ deviated clearly. The binding state of oxygen on this surface at $80 \mathrm{~K}$ appeared to be different from that on the same surface at $300 \mathrm{~K}$.

\section{Introduction}

In the last few years the $\mathrm{Si}(100)$ surface has been the subject of a number of theoretical and experimental studies. With advanced computer techniques it has now become possible to calculate the density of states as a function of energy in the neighbourhood of a surfacc, not only for ideal but also for simply reconstructed surfaces. This was carried out for the $\mathrm{Si}(100)$ surface [1-4], for which a $(2 \times 1)$ structure has been found in several LEED experiments [5-7]. Structural models were tested by comparing experimental data from UPS and/or optical measurements (such as ellipsometry and reflectometry) with band structure calculations and fitting the results of dynamical calculations of LEED intensities [8-11] to measured intensity-voltage spectra. For $\mathrm{Si}(100)$ surfaces at $300 \mathrm{~K}$ several surface structures have been reported $[12,13]$. Up to now no detailed band structure calculations have been made for the reconstructed $\mathrm{Si}(110)$ surface.

In continuation of earlier results on the clean $\operatorname{Si}(111) 7 \times 7$ surface [14], we carried out differential reflection measurements on clean $\mathrm{Si}(100)$ and (110) at 300 and $80 \mathrm{~K}$. Comparison of the sample characteristics before and after adsorption of 
an oxygen monolayer presented information about optical transitions due to surface states at the clean surface. The results were collated with band structure calculations for the $\mathrm{Si}(100)$ reconstructed surface. The oxygen adsorption kinetics on the (100) and (110) surfaces at 300 and $80 \mathrm{~K}$ were also investigated and compared with the results for $\mathrm{Si}(111) 7 \times 7$ [14].

\section{Experimental}

The silicon samples $(10 \times 30 \times 0.2 \mathrm{~mm})$ used were made out of $10 \Omega \mathrm{cm}$-type boron-doped crystals $\left((100)\right.$ and $(110)$ orientation within $\left.0.5^{\circ}\right)$ purchased from Siltronix. The measuring procedures and the UHV system, used for the experiments, have been previously described [14].

The measurements on the $\mathrm{Si}(100)$ surfaces were carried out with the differential reflectometer of which the description was given in ref. [14]. The electronic part of this reflectometer was an analog system, in which a lock-in amplifier was employed for handling the detector signal. With this system, we could not scan the wavelength during a measurement because of the relatively long integration times needed for sufficient sensitivity and the long-term drift of the analog devices. To solve this problem we automatized the electronic part of the reflectometer with the help of a microprocessor. The monochromator is also controlled by the processor. It is thus possible to scan the wavelength automatically during a reflection experiment. Details of this system will be described elsewhere [15]. The sensitivity of the automatic differential reflectometer in the photon energy range of 1.0 to $3.0 \mathrm{eV}$ in terms of the relative difference in reflectivity $\Delta R / R$ is $1 \times 10^{-4}$. The long-term stability during periods of $12 \mathrm{~h}$ and longer is $3 \times 10^{-4}$. Earlier results on Si(111) $7 \times 7$ [14] were checked by the automatic reflectometer in the photon energy range of 1.5 to $3.0 \mathrm{eV}$. Agreement was found within experimental error.

\section{Surface states at the clean Si(100) and (110) surfaces}

\subsection{Reflectivity measurements}

The surface contribution was determined from the data by measuring the relative difference in reflectivity [16]

$$
\frac{\Delta R}{R}=\frac{R_{\text {clean }}-R_{\text {ox }}}{R_{\text {clean }}},
$$

between a thermally cleaned silicon surface, containing numerous surface states $\left(\approx 10^{15} \mathrm{~cm}^{-2}\right)$ and the same surface covered with a monolayer of oxygen and a drastically decreased number of states $\left(\approx 10^{12} \mathrm{~cm}^{-2}\right)$. The measurements on the $\mathrm{Si}(100)$ surfaces were performed with the manual reflectometer at photon energies 

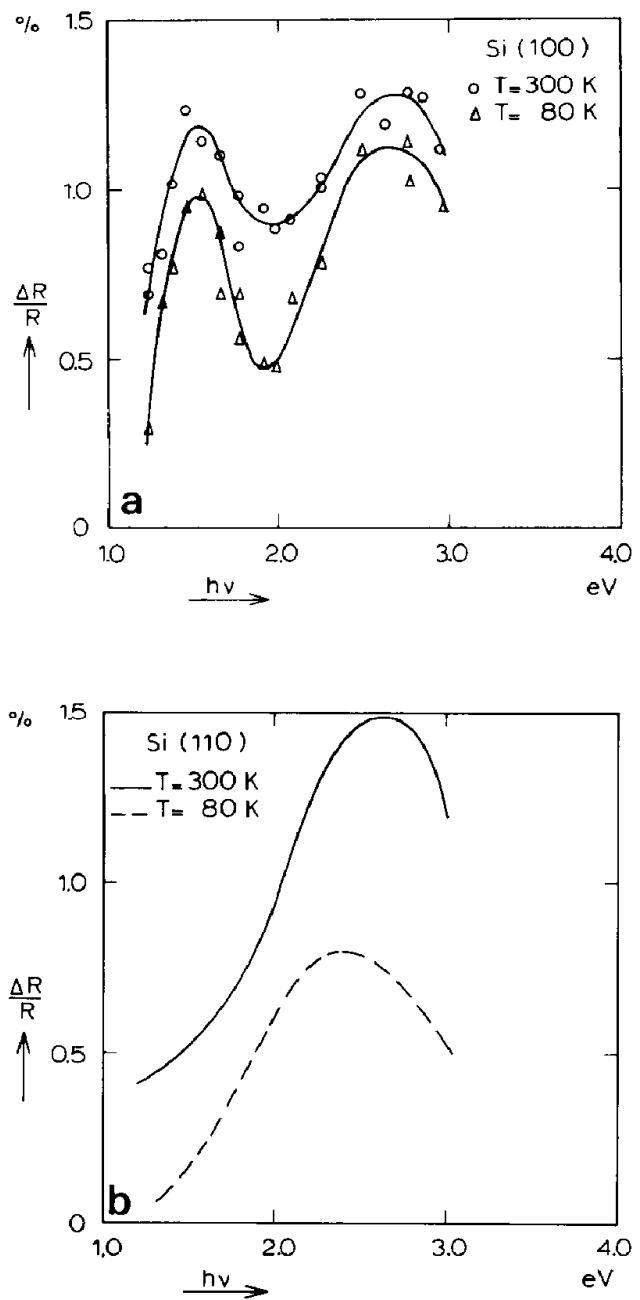

Fig. 1 . The relative difference in reflectivity $\Delta R / R$ before and after adsorption of a monolayer of oxygen on the clean $\mathrm{Si}(100)$ (a), and (110) surface (b) as a function of the photon energy $h \nu$ at 300 and $80 \mathrm{~K}$.

of 1.0 to $3.0 \mathrm{eV}$ at intervals of approximately $0.1 \mathrm{eV}$. The wavelength dependent data for the $\mathrm{Si}(110)$ surfaces were recorded practically continuously with the automatic reflectometer. The results of several experiments on two different $\mathrm{Si}(100)$ sample surfaces were averaged and are given in fig. 1a. Fig. $1 \mathrm{~b}$ shows the results for the $\mathrm{Si}(110)$ surface. The curves were obtained by averaging several measurements on the same sample. 


\subsection{Surface state transitions}

As for $\operatorname{Si}(111) 7 \times 7$ [14], the dielectric layer model of Meyer et al. [17] was used for the interpretation of the reflectometric data. In this model, the electronic states, characteristic for the clean surface region, are represented by an absorbing layer of complex dielectric constant $\hat{\epsilon}_{\mathrm{ss}}$ and thickness $d_{\mathrm{ss}}$, which is much smaller than the wavelength $\lambda$ of the incident light beam. It is assumed that oxygen adsorption replaces the surface states layer on the bulk substrate $\left(\hat{\epsilon}_{\mathrm{b}}\right)$ by an adsorbed layer of oxygen $\left(\hat{c}_{o x}\right)$. The imaginary part of the complex dielectric constant of the surface states layer was calculated from the measured values of $\Delta R / R$ by using the relation of McIntyre and Aspnes [18] at normal incidence:

$\frac{\Delta R}{R}=-\frac{8 \pi d_{\mathrm{ss}}}{\lambda} \operatorname{Im}\left(\frac{\hat{\epsilon}_{\mathrm{ss}}-\hat{\epsilon}_{\mathrm{b}}}{1-\hat{\epsilon}_{\mathrm{b}}}\right)$,

since the influence of the oxygen layer on $\Delta R / R$ in the energy range of 1.0 to $3.0 \mathrm{eV}$ was calculated to be smaller than $2 \times 10^{-4}$ and thus negligible. Analogous to Meyer et al. [17], we took $d_{\mathrm{ss}}=0.5 \mathrm{~nm}$. In figs. $2 \mathrm{a}$ and $2 \mathrm{~b}$ the results of the calculations are given for the $\mathrm{Si}(100)$ and $(110)$ surfaces, respectively. The room temperature curve of fig. $2 \mathrm{a}$ shows two peaks, which sharpen at $80 \mathrm{~K}$. At $80 \mathrm{~K}$ the heights of the peaks were approximately $15 \%$ smaller than at $300 \mathrm{~K}$. For the $\mathrm{Si}(110)$ surface at $300 \mathrm{~K}$ only one peak was found in the energy range studied. In contrast to expectations based on the results for the $\mathrm{Si}(100)$ and (111) $7 \times 7$ surfaces and on theoretical speculations, this peak did not clearly sharpen at $80 \mathrm{~K}$. However, the height of the peak diminished by a factor of two with respect to the value at $300 \mathrm{~K}$. The room temperature results for $\mathrm{Si}(100)$ and $(110)$ are in reasonable agreement with the ellipsometric data of Meyer et al. [17,19].

The reflectometric data give information about optical transitions between filled and empty electronic states, in which at least one type of states is characteristic for the sample surface. The joint density of states, which is proportional to $(h \nu)^{2}$ $\operatorname{Im}\left(\hat{\epsilon}_{\mathrm{ss}}-\hat{\epsilon}_{\mathrm{b}}\right)$, can be calculated from the curves of figs. $2 \mathrm{a}$ and $2 \mathrm{~b}$. The maxima in the joint density of states for the $\mathrm{Si}(100)$ surface occurred at $3.0 \pm 0.2 \mathrm{eV}$ and $1.60 \pm 0.05 \mathrm{eV}$, both at 300 and $80 \mathrm{~K}$; that at $3.0 \mathrm{eV}$ was found by extrapolation. The maximum for the $\mathrm{Si}(110)$ surface could also be localized at $3.0 \pm 0.2 \mathrm{eV}$. The maxima for the $\mathrm{Si}(111) 7 \times 7$ surface were positioned at $2.9 \pm 0.1 \mathrm{eV}$ and $1.76 \pm$ $0.04 \mathrm{eV}$ [14]. Within experimental error the position and height of the main peak at $3.0 \mathrm{eV}$ appears to be the same for all measured silicon surfaces at room temperature within experimental error. For a good comparison the heights of the peaks were corrected for the orientation dependent number of surface atoms per unit surface area. The peaks at $3 \mathrm{eV}$ can be attributed to optical transitions from a surface states band near the top of the valence band to bulk conduction band levels [20]. In contrast, the position of the second peak is strongly dependent on the orientation and structure of the surface. For the (110) surface a second peak was neither found in the photon energy range of 1.0 to $3.0 \mathrm{eV}$, nor in the infrared 

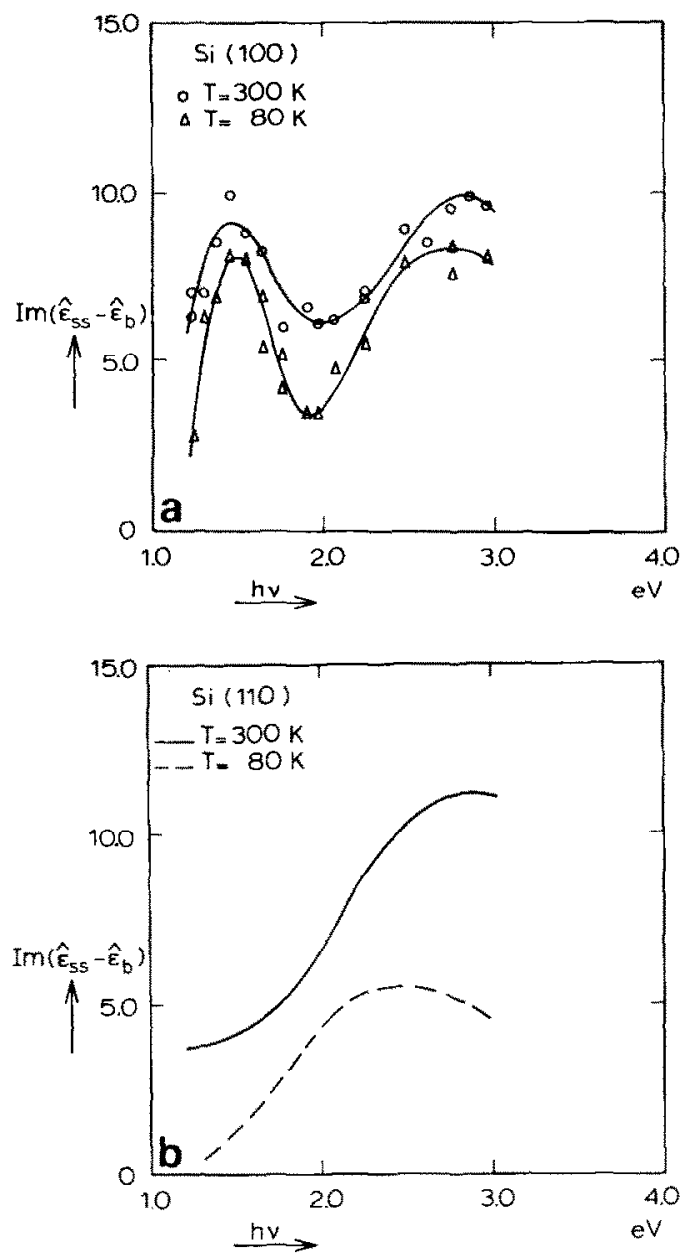

Fig. 2. The imaginary part of the difference in complex dielectric constant $\operatorname{Im}\left(\hat{\epsilon}_{\mathrm{SS}} \hat{\epsilon}_{\mathfrak{b}}\right)$ between the surface states layer and the bulk substrate of the (100) (a), and (110) surface (b) as a function of the photon energy $h v$ at 300 and $80 \mathrm{~K}$.

region down to $0.5 \mathrm{eV}$, as for the cleaved $\mathrm{Si}(111) 2 \times 1$ surface [21]. Earlier LEED experiments on clean $\mathrm{Si}(110)$ surfaces revealed several possible surface structures at room temperature $[12,13]$. In our experiments we were not able to check the structure of the clean (110) surface by LEED. After a heating period of 20-30 s at $1150^{\circ} \mathrm{C}$ our samples were rapidly cooled. This has probably resulted in a $(5 \times 1)$ structure at $300 \mathrm{~K}[13]$. Experiments on slowly cooled samples, giving another surface structure according to Olshanetsky et al. [13], gave the same results within experimental error. 
In chemical adsorption experiments on clean semiconductor surfaces the band bending at the surface is expected to be changed as a result of a charge redistribution. This might cause a change in the optical parameters due to the Franz-Keldysh effect [22]. From electroreflectance experiments [23], the critical points in the bulk optical structure for silicon were found to be positioned above $3 \mathrm{eV}$. Therefore, electric field effects will not have a noticeable influence on the shape of the above-mentioned curves in the photonenergy region up to $3.0 \mathrm{eV}$.

\subsection{Structural models for the Si(100) surface}

With LEED [5-7,24] and He diffraction measurements [25], two superstructures have been observed for clean $\mathrm{Si}(100)$ surfaces, namely $(2 \times 1)$ and a $\mathrm{c}(4 \times 2)$ structure. A number of models has been proposed for the $(2 \times 1)$ structure of the $\mathrm{Si}(100)$ surface; a review is given in table 1 . Also shown is the extent of agreement of the model calculations with LEED data and integral and angular resolved photoemission spectra. The symmetric dimer models are in good agreement with integral

Table 1

Structural models for the Si(100) surface

Extent of agreement between model and experiment

\begin{tabular}{llll}
\cline { 2 - 4 } Experiment & LEED & UPS & Angular resolved \\
& {$[5-7,24]$} & {$[31]$} & UPS [32]
\end{tabular}

Model with

Surface

structure

1. Vacancies

$2 \times 1$

Bad [8]

Bad [1] Bad [3]

$[5,26,29]$

2. Conjugated chains

$[8,28]$

$2 \times 1$

Reasonable

Bad [4] Bad [3]

3. Overlayers

$2 \times 1$

[8]

[30]

4. Dimers

[5,27]

5. Symmetric dimers

with subsurface

distortions [34]

Reasonable

[10]

$2 \times 1$

Bad [8]

Good

Bad [3]

$[1,2,33]$

Good

Bad [3]

[10,11,34], reasonable [9]

6. Asymmetric dimers

$2 \times 1$

with subsurface

$4 \times 2$

distortions [3]

7. Multilayer

vacancies

$4 \times 2$

Reasonable

$[11,24]$ 
photoemission experiments [31], but give a metallic surface electronic structure which is in disagreement with angular resolved photoemission experiments [32]. In order to fit the dynamical model calculations for the $(2 \times 1)$ structures to the measured LEED spectra, subsurface distortions have to be introduced. The asymmetric or ionic dimer model [3] results in a semiconductor surface electronic structure with a gap of approximately $0.8 \mathrm{eV}$. Up to now this appears to be the only model that is in reasonable agreement with all experimental results. The highest filled surface states for the five-layer relaxed ionic dimer model form a band from approximately 1.3 to $0.2 \mathrm{eV}$ below the bulk valence band maximum, whereas a band of empty surface states is formed from approximately 0.6 to $1.0 \mathrm{eV}$ above it.

The asymmetric dimer model is also in qualitative agreement with our present reflectometric data and earlier ellipsometric results [19]. The high-energy peak in the curves of fig. 3 can be assigned to optical transitions from the filled surface states band near the top of the valence band to empty bulk conduction band levels in the same way as was proposed for the main peak from the reflectometric experiments on the $\mathrm{Si}(111) 7 \times 7$ surface [14]. The low-energy peak may be attributed to transitions to an empty surface states band in the gap region. The maximum in the joint density of states at $1.6 \mathrm{eV}$ agrees with the above-mentioned band structure calculations for the asymmetric dimer model.

\section{Effects of oxygen adsorption at 300 and $80 \mathrm{~K}$}

\subsection{Si(100)}

The disappearance of the high-energy peak at 300 and $80 \mathrm{~K}$ upon oxygen pressure is shown in fig. 3a. The curves were obtained by averaging the results of several adsorption experiments on two different samples at oxygen inlet pressures in the range of $10^{-8}$ to $10^{-7}$ Torr. They show a linear increase of $\Delta R / R$ as a function of the oxygen exposure up to $80-90 \%$ of the saturation coverage. The sticking coefficients in this region appeared to be independent of the oxygen pressure. During the adsorption experiments, the ion pump was in operation, but the ionization gauge was turned off. Control experiments showed that for oxygen pressures lower than $2 \times 10^{-7}$ Torr, the pressure remained constant for the duration of the experiment. As a result, this could be measured afterwards. Replacing the ion pump by a turbomolecular pump, appeared to have no significant influence on the adsorption kinetics of oxygen on the $\mathrm{Si}(100)$ surface.

The saturation coverage is assumed to be the same as for the (111) surface [35], being one oxygen atom per silicon surface atom. This assumption is favoured by earlier results with LEED, UPS, ELS [36] and AES [37], which showed no significant differences between the (111), (100) and (110)-silicon surfaces, covered with oxygen. The sticking coefficient $(s)$ was determined with:

$$
\frac{\mathrm{d}}{\mathrm{d} t} \theta=s(\theta) \frac{\nu_{\mathrm{O}} p}{N_{\mathrm{o}}},
$$


where $p$ is the pressure in Torr, $t$ the exposure time, $\theta$ the fractional coverage, $\nu_{0}$ the number of molecules that strike a unit area of the surface per unit time and unit pressure $\left(\nu_{0}=3.48 \times 10^{20} / \mathrm{cm}^{2} \cdot \mathrm{s} \cdot\right.$ Torr for oxygen molecules at $\left.300 \mathrm{~K}\right)$ and $N_{0}$ the number of surface sites for a molecule $\left(N_{0}=3.39 \times 10^{14} / \mathrm{cm}^{2}\right.$ for $\left.\operatorname{Si}(100)\right)$. The sticking coefficient up to $80 \%$ of the saturation coverage at $300 \mathrm{~K}$ was calculated to be :

$s=0.036 \pm 0.004$.

This values is in reasonable agreement with earlier values from LEED $(s=0.02$ [5]) and ellipsometry $(s=0.02$ [37]). For the $\mathrm{Si}(111) 7 \times 7$ surface at $300 \mathrm{~K}$ we calculated a sticking coefficient of the same order of magnitude $(s=0.08$ [14]).

From the curves of fig. 3a it appears that at $80 \mathrm{~K}$ the oxygen adsorption on $\mathrm{Si}(100)$ takes place about twice as fast as at $300 \mathrm{~K}$. This is in qualitative agreement with the results for the $\mathrm{Si}(111) 7 \times 7$ surface [14]. As has also been found by ELS measurements [36] the adsorption kinetics for oxygen on $\mathrm{Si}(100)$ and $\mathrm{Si}(111)$ $7 \times 7$ are very similar.

\section{2. $\operatorname{Si}(110)$}

The disappearance of the peak for the $\mathrm{Si}(110)$ surface at 300 and $80 \mathrm{~K}$ upon exposure to oxygen is shown in fig. $3 \mathrm{~b}$. The curves were obtained by averaging the results of several measurements on the same sample at different oxygen inlet pressures $\left(10^{-8}\right.$ to $10^{-7}$ Torr $)$. Again assuming a saturation coverage of one oxygen atom per silicon surface atom, the sticking coefficient up to $80-90 \%$ of the saturation coverage (linear part of the curve) for oxygen adsorption on the rapidly cooled $\mathrm{Si}(110)$ surface at $300 \mathrm{~K}$ appeared to be:

$s=0.028 \pm 0.004$.

This value is of the same order of magnitude as those found for the $\mathrm{Si}(100)$ and (111) $7 \times 7$ surfaces. The room temperature behaviour of the three surfaces with respect to oxygen adsorption appears to be similar. However, the (110) surfaces deviates clearly at $80 \mathrm{~K}$. In contrast to the (111) $7 \times 7$ and (100) surfaces, the oxygen adsorption on thermally cleaned $\mathrm{Si}(110)$ at $80 \mathrm{~K}$ proceeds more slowly than at $300 \mathrm{~K}$. In addition, $\Delta R / R$ at saturation coverage is approximately half that at room temperature. In order to obtain more information about the nature of the low-temperature effects an additional experiment was carried out.

Having reached the saturation value for $\Delta R / R$ at $80 \mathrm{~K}$ in an adsorption experiment, oxygen inlet was stopped and the pressure was reduced down to approximately $10^{-9}$ Torr without observing a change in $\Delta R / R$. Subsequently the sample was heated resistively to $300 \mathrm{~K}$ for a few minutes and rapidly cooled to $80 \mathrm{~K}$ again. After a stabilization period, $\Delta R / R$ appeared to have increased compared to the situation before the heating cycle. A second heating cycle caused no further significant change. The new saturation value for $\Delta R / R$ was approximately equal to that found 

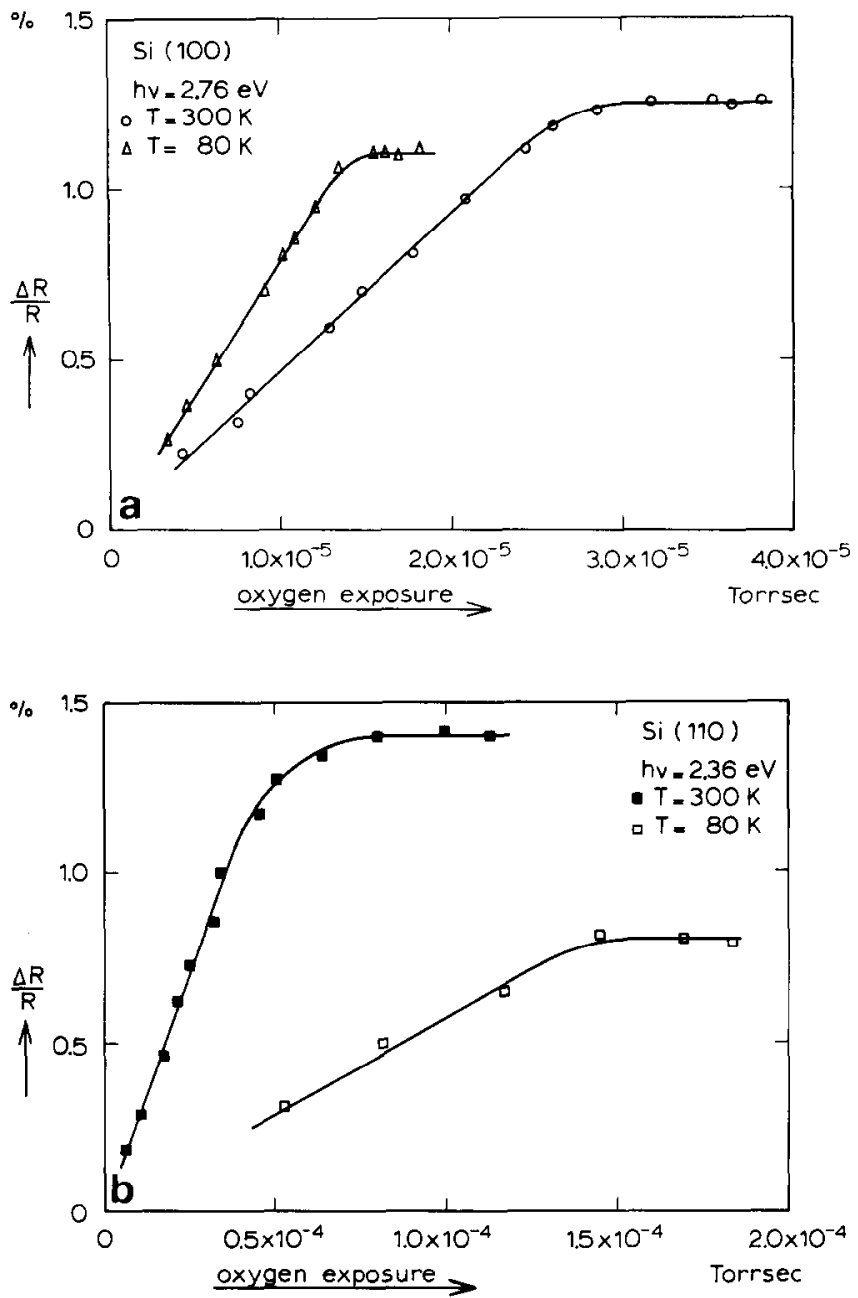

Fig. 3. The relative change in reflectivity $\Delta R / R$ versus oxygen exposure for $\operatorname{Si}(100)$ (a), and $\mathrm{Si}(110)(\mathrm{b})$ at fixed photon energies at 300 and $80 \mathrm{~K}$.

in the adsorption experiments at $300 \mathrm{~K}$. A heating period of a few minutes at a base pressure of $10^{-9}$ Torr (oxygen partial pressure $<5 \times 10^{-10}$ Torr) is too short to cause nuticeable exlra oxygen adsorption during the heating cycle at $300 \mathrm{~K}$ with a concomitant change in $\Delta R / R$. We therefore conclude that the binding state of oxygen on $\mathrm{Si}(110)$ at $80 \mathrm{~K}$ is different from that at $300 \mathrm{~K}$ and that the saturation coverage is the same. The irreversible transition from the low-temperature state to the room temperature state can be achieved by raising the temperature of the sample during a few minutes. 


\section{Conclusions}

From the present reflectometric study of surface state transitions and oxygen adsorption on clean $\mathrm{Si}(100)$ and (110) surfaces and earlier results for the $\mathrm{Si}(111)$ $7 \times 7$ surface we may conclude:

(a) The position of the main peak at $3.0 \mathrm{eV}$ is the same for the $\mathrm{Si}(111) 7 \times 7,(100)$ and (110) surfaces. This is also true for the corrected height at $300 \mathrm{~K}$. At $80 \mathrm{~K}$ the $\mathrm{Si}(110)$ surface deviates clearly; the measured peak height at that temperature diminished by a factor of two with respect to the value at $300 \mathrm{~K}$.

(b) The position of the second peak is strongly dependent on the orientation and structure of the surface. For the $\mathrm{Si}(110)$ surface no second peak was found in the photon energy region from 0.5 to $3.0 \mathrm{eV}$.

(c) The sticking coefficients for oxygen adsorption on all measured surfaces appeared to remain constant up to coverages in the order of $80-90 \%$ of a monolayer. This is in favour of a model, in which the oxygen adsorbs in a weakly bound mobile precursor state $[38,39]$, before it is chemisorbed on the surface. The sticking coefficients at $300 \mathrm{~K}$ are of the same order of magnitude. At $80 \mathrm{~K}$, the sticking coefficients for the chemisorption of oxygen on the (111) $7 \times 7$ and (100) surfaces appeared to increase with respect to the values at $300 \mathrm{~K}$. Again, the behaviour of the (110) surface deviated clearly. At $80 \mathrm{~K}$, oxygen adsorbed in a low-temperature binding state which differed from that at $300 \mathrm{~K}$.

\section{Acknowledgements}

The authors wish to thank Professor G.A. Bootsma for his critical reading of the manuscript and Dr. A.J. van Bommel, Philips, Eindhoven (The Netherlands), for valuable discussions.

\section{References}

[1] J.A. Appelbaum, G.A. Baraff and D.R. Hamann, Phys. Rev. B14 (1976) 588.

[2] I.B. Ortenburger, S. Ciraci and I.P. Batra, J. Phys. C9 (1976) 4185.

[3] D.J. Chadi, Phys. Rev. Letters 43 (1979) 43.

[4] G.P. Kerker, S.G. Louie and M.L. Cohen, Phys. Rev. B17 (1978) 706.

[5] R.E. Schlier and H.E. Farnsworth, J. Chem. Phys. 30 (1959) 917.

[6] J.J. Lander and J. Morrison, J. Chem. Phys. 37 (1962) 729.

[7] A. Ignatiev, F. Jona, M. Debe, D.E. Johnson, S.J. White and D.P. Woodruff, J. Phys. C10 (1977) 1109.

[8] F. Jona, H.D. Shih, A. Ignatiev, D.W. Jepsen and P.M. Marcus, J. Phys. C10 (1977) L67.

[9] F. Jona, H.D. Shih, D.W. Jepsen and P.M. Marcus, Bull. Am. Phys. Soc. 24 (1979) 467;

F. Jona, H.D. Shih, D.W. Jepsen and P.M. Marcus, J. Phys. C12 (1979) L455.

[10] K.A.R. Mitchell and M.A. Van Hove, Surface Sci. 75 (1978) L147.

[11] S.Y. Tong and A.L. Maldonado, Surface Sci. 78 (1978) 459. 
[12] F. Jona, IBM J. Res. Develop. 9 (1965) 375.

[13] B.Z. Olshanetsky and A.A. Shklyaev, Surface Sci. 67 (1977) 581.

[14] P.E. Wierenga, A. van Silfhout and M.J. Sparnaay, Surface Sci. 87 (1979) 43.

[15] K.O.v.d. Werf and P.E. Wierenga, to be published.

[16] In ref. [14], the sign in the definition of $\Delta R$ has to be changed.

[17] F. Meyer, E.E. de Kluizenaar and G.A. Bootsma, Surface Sci. 27 (1971) 88.

[18] J.D.E. McIntyre and D.E. Aspnes, Surface Sci. 24 (1971) 417.

[19] F. Meyer, Surface Sci. 56 (1976) 37.

[20] J.E. Rowe, H. Ibach and H. Froitzheim, Surface Sci. 48 (1975) 44.

[21] G. Chiarotti, S. Nannarone, R. Pastore and P. Chiaradia, Phys. Rev. B4 (1971) 3398.

[22] R. Dorn and H. Lüth, Phys. Rev. Letters 33 (1974) 1024;

F. Lukes, Surface Sci. 49 (1975) 344;

R. Matz and H. Luith, Appl. Phys. 18 (1979) 123.

[23] M. Cardona, K.L. Shaklee and F.H. Pollak, Phys. Rev. 154 (1967) 696.

[24] T.D. Poppendieck, T.C. Ngoc and M.B. Webb, Surface Sci. 75 (1978) 287.

[25] M.J. Cardillo and G.E. Becker, Phys. Rev. Letters 40 (1978) 1148.

[26] J.C. Phillips, Surface Sci. 40 (1973) 459.

[27] J.D. Levine, Surface Sci. 34 (1973) 90.

[28] R. Seiwatz, Surface Sci. 2 (1964) 473.

[29] W.A. Harrison, Surface Sci. 55 (1976) 1.

[30] K.A.R. Mitchell, Surface Sci. 64 (1977) 797.

[31] J.E. Rowe and H. Ibach, Phys. Rev. Letters 32 (1974) 421.

[32] F.J. Himpsel and D.E. Eastman, 6th Ann. Conf. on Physics of Compound Semiconductor Interfaces, Asilomar, CA, Contribution 44.

[33] J.A. Appelbaum, G.A. Baraff and D.R. Hamann, Phys. Rev. B15 (1977) 2408.

[34] J.A. Appelbaum and D.R. Hamann, Surface Sci. 74 (1978) 21.

[35] A.H. Boonstra, Philips Res. Rept. Suppl. No. 3 (1968).

[36] H. Ibach and J.E. Rowe, Phys. Rev. B9 (1974) 1951; B10 (1974) 710.

[37] F. Meyer and J.J. Vrakking, Surface Sci. 38 (1973) 275.

[38] C. Kohrt and R. Gomer, J. Chem. Phys. 52 (1970) 3283.

[39] D.A. King and M.G. Wells, Proc. Roy. Soc. (London) A339 (1974) 245. 